\title{
Agathomyia Wankowiczii Schnabl und aurantiaca Bezzi nebst einer Übersicht der europ. Agathomyia-Arten (Dipt.).
}

Von P. Leander Czerny in Kremsmünster, Oberösterreich.

Dr. J. Schnabl beschrieb im Pamietnik Fizyjograf. 1884, pag. 293 (Warschau) unter dem Namen Callomyia Wankowicriı q eine aus dem Gouvernement Mohilew stammende, durch ihre orangegelbe Färbung von allen bis dahin bekannten Callimyia-Arten ${ }^{1}$ ) verschiedene Art, deren lateinische Diagnose - die Charakteristik ist polnisch gegeben - lautet: »Aurantiaca, antennis, scutello, halteribusque concoloribus; alis flavescentibus«.

Neun Jahre später beschrieb Prof. Dr. M. Bezzi in dieser Zeitung, Jahrg. XII, 1903, pag. 304 unter dem Namen Callomyia aurantiaca ein auf dem Monte Baldo bei Mori gefangenes Männchen, dessen Diagnose lautet: »Aurantiaca, capite, thoracis lateribus, pectore, metanoto ultimoque abdominis segmento atris; antennis pedibusque luteis, illis articulo tertio subulato; alis hyalinis, nervis, costa excepta, lutescentibus. Long. $3 \mathrm{~mm}$ (exsiccata) «.

Diese beiden Arten wurden von Fr. Hendel in dieser Zeitung Jahrg. XXI, 1902, pag. 265, Nr. 23, für eine Art erklärt.

Prof. Bezzi erwiderte im Jahrg. XXII. 1903, pag. 21 und 22 dieser Zeitung auf die von Hendel aufgestellte Synonymie, indem er den Unterschied beider Arten in der Körper- und Flügelfärbung hervorhob, der nicht bloß vom Geschlechte herstammen könnte, gab aber die Wahrscheinlichkeit der Synonymie zu. Zugleich teilte er mit, daß bei der Schnablschen Art ebenso wie bei seiner das dritte Fühlerglied verlängert und die erste Längsader nicht beborstet sei und daher beide Arten zu der Verrallschen Gattung Agathomyia gehörten.

Ich bin nun in der angenehmen Lage erklären zu können, daß die Bezzische Art mit der Schnablschen nicht identisch ist. Am 17. Juni d. J. fing ich in der Nähe von Kremsmünster bei Kremsursprung ein Agathomyia-Männchen, das zweifelsohne die A. Wankowicrii ist.

Da die polnische Beschreibung der Agathomyia Wankowiczii nur wenigen Dipterologen verständlich sein dürfte, so lasse ich eine Beschreibung meines Männchens folgen.

1) Callimyia, em. Verrall, British Flies, Vol. VIII, 1901, p. 24.

Wiener Entomologisehe Zeitung, XXIII. Jahrg., Heft VII (31. August 1904). 
Das ganze Tier ist orangegelb, nur die Stirn, das Gesicht, die Backen, der Scheitel, der Hinterkopf und der hintere Teil des eingeschlagenen Hypopygs sind schwarz. Die Postokularcilien sind orangegelb, die oberen scheinen schwarz zu sein, schimmern aber in gewisser Richtung orangegelb. Die Augenfacetten der oberen Hälfte sind größer als die der unteren. Das zweite Fühlerglied trägt oben eine gelbe Borste, das dritte ist nicht so lang ausgezogen wie bei antemnata Ztt., es ist ungefähr nur zweimal so lang als breit; die Fühlerborste erscheint in gewisser Richtung dunkel und ist mehr als zweimal so lang als das dritte Fühlerglied. Beborstung des Thorax: eine Reihe sehr kleiner, weit vor dem Vorder- und Hinterrande aufhörender Akrostichalborsten, eine hintere Dorsocentralborste und von dieser aus eine vorn zu den Schultern abbiegende Reihe kleiner Borsten, zwei Supraalarborsten, fünf in schiefer Reihe stehende Präalarborsten, acht Skutellarborsten, zwei längere an der Spitze und je drei kürzere an den Seiten. Alle diese Borsten sind schwarz. Beine: Vorderschenkel auf der Rückseite unten, nahe der Basis mit dem gewöhnlichen gebogenen Dorne, Vorderschienen nahe an der Spitze mit einer spornartigen Borste; Mittelschenkel auf der Vorderseite zwischen der Mitte und der Spitze mit einer kleinen Schwiele, Mittelschienen im Wurzeldrittel mit einer Borste und an der Spitze innen mit einem Sporne, Metatarsus der Mittelbeine mit zwei Borsten, von denen die kürzere ganz nahe an der Basis, die längere etwas entfernter davon steht; Hinterschenkel rückwärts auf der Unterseite nahe an der Basis mit einer starken Borste, oben mit einer Borstenreihe, Metatarsus der Hinterbeine mit einer kleinen Borste unten nahe an der Wurzel. Alle diese Dorne und Borsten sind bräunlich orangegelb. Flügel irisierend, gelb, die Adern stärker gelb, die hintere Querader unten schief nach außen gestellt. Schüppchen schwarz gerandet und schwarz gewimpert. Borsten des Hinterleibes sehr spärlich, kurz und schwarz, vorn an den Seiten einige gelbe Borsten.

Übersicht der europäischen Agathomyia-Arten. ${ }^{1}$ )

A. Männchen.

1. Thorax ganz oder zum Teil orangegelb

Thorax schwarz oder grau

2. Thorax ganz orangegelb

Wankowic:ii Schnabl

1) Nach Verralls Catalogue of the Platyperidae of the European District 1900, pag. 4 und 5 ist A. Megerlei Mg. synonym mit Falléni Ztt., cinerea Ztt. und Hoffmanseggii Mg. mit antennata Ztt. - Ich kenne nur antennata Ztt. ठ , Falleni Ztt. \& und Wanlowicrii Schnabl ơ, alle drei aus hiesiger Gegend. 
Thorax vorn, an den Seiten, auf der Brust und am Hinterrücken schwarz . . . aurantiaca Bezzi

3. Thorax dunkelgrau, Hinterleib gelb, mit braunen Flecken . . . . . . . Fallémi Ztt.

Thorax und Hinterleib schwarz . . . . . . . . . . . 4

4. Mittelschienen im Wurzeldrittel mit einer Borste, Metatarsus der Mittelbeine mit einer oder zwei Borsten ganz nahe an der Wurzel . . . . . . . . . antennata Ztt.

Mittelschienen ohne Borste, Metatarsus der Mittelbeine mit einer Borste im Wurzeldrittel . . Collini Verr.

B. Weibchen:

1. Thorax orangegelb . . . . . . . . Wankowiczii Schnabl Thorax schwarz oder grau . . . . . . . . . . . . . . 2

2. Hinterleib zum Teile orangegelb oder braun, mit gelben Hinterrandsäumen . . . . . . . . . . 3 Hinterleib anders gefärbt . . . . . . . . . . . . 4

3. Die ersten drei Segmente des Hinterleibes und das vierte mit Ausnahme eines schwarzen Seitenfleckes orangegelb . . . Zetterstedti Whlbg. Hinterleib braun, mit gelben Hinterrandsäumen . . . . . . . . . Falléni Ztt.

4. Hinterleib ganz schwarz . . . . . . viduella Ztt. Hinterleib nicht ganz schwarz . . . . . . . . . . . 5

5. Erstes und zweites Segment des Hinterleibes an den Seiten und die beiden letzten Segmente silberfarbig, drittes bis fünftes Segment schwarz . . . . Collini Verr.

Hinterleib schwarz, mit grauen Flecken auf dem Rücken des ersten und zweiten und zum Teile des dritten Segmentes oder fast alle vier Basalsegmente grau, mit dreieckigen schwarzen Rückenflecken . antennata Ztt. 


\section{$2 \mathrm{BHL}$ Biodiversity Heritage Library}

Czerny, Leander. 1904. "Agathomyia Wankowiczii SCHNABL und aurantiaca BEZZI nebst einer Übersicht der europ. Agathomyia-Arten (Dipt.)." Wiener entomologische Zeitung 23, 137-139. https://doi.org/10.5962/bhl.part.27196.

View This Item Online: https://www.biodiversitylibrary.org/item/44102

DOI: https://doi.org/10.5962/bhl.part.27196

Permalink: https://www.biodiversitylibrary.org/partpdf/27196

\section{Holding Institution}

Smithsonian Libraries

\section{Sponsored by}

Smithsonian

\section{Copyright \& Reuse}

Copyright Status: NOT_IN_COPYRIGHT

This document was created from content at the Biodiversity Heritage Library, the world's largest open access digital library for biodiversity literature and archives. Visit BHL at https://www.biodiversitylibrary.org. 